\title{
Short Frame Length Approximation for IRSA
}

\author{
M. Çağatay Moroğlu, H. Murat Gürsu, Student Member, IEEE, Federico \\ Clazzer, Member, IEEE, and Wolfgang Kellerer, Senior Member, IEEE
}

\begin{abstract}
In this letter, we propose a short-frame (SF) approximation for the packet loss rate of Irregular Repetition Slotted ALOHA (IRSA) particularly suitable for very short frames, up to 50 slots. The reason for limiting the frame size is to allow low-latency communications, a typical requirement in Industrial Internet of Things (IIoT) applications. We extend the approximation of [1] (named EF) through a recursive evaluation of inter-related stopping sets (STs). The tighter approximation yields the user degree distribution that minimizes the packet loss rate (PLR). For a scenario with 10 slots and 5 users, the degree distribution optimized adopting the SF approximation results in a $20 \%$ lower PLR compared to the one optimized with the EF approximation.
\end{abstract}

\section{Index Terms}

Finite Length Analysis, Packet Loss Rate, Random Access

\section{INTRODUCTION}

The upcoming industrial revolution will be triggered by Industrial Internet of Things (IIoT) where a plurality of inter-connected devices produce and collect data. IIoT devices have sporadic activity that requires small packets to be transmitted within a limited time budget in a highly reliable way [2]. Therefore, IIoT communications should support low latency and high reliability. One obstacle for providing low initial-access latency is the signaling overhead, which can be

M. Ç. Moroğlu, H. M. Gürsu and W. Kellerer are with the Chair of Communication Networks, Technische Universität München, Theresienstr. 90, 80333 Munich, Germany.

F. Clazzer is with the Inst. of Communications and Navigation, German Aerospace Center (DLR), Oberpfaffenhofen, Germany (e-mail: federico.clazzer@dlr.de),

“(C2020 IEEE. Personal use of this material is permitted. Permission from IEEE must be obtained for all other uses, in any current or future media, including reprinting/republishing this material for advertising or promotional purposes, creating new collective works, for resale or redistribution to servers or lists, or reuse of any copyrighted component of this work in other works. DOI: 10.1109/LWC.2020.3008814 
avoided with grant-free access protocols. Currently deployed grant-free algorithms solve the reliability problem with over-provisioning in the 5G New Radio (NR) [3]. Modern random access algorithms instead avoid over-provisioning through the use of Interference Cancellation (IC) [4]. A recent work [5] has discussed their adaptation to 5G without reliability guarantees. Irregular Repetition Slotted ALOHA (IRSA) is one popular modern random access algorithm. It can reach a normalized throughput of 0.97 packets/slot [6] for very large frames and 0.8 with 1000 slots. However, achieving low latency requires short IRSA frames, i.e. 10 to 50 slots. In this regime the asymptotic analysis of [6] becomes loose, and different strategies to model the performance shall be followed. Note that a precise characterization of the packet loss rate (PLR) in IRSA for finite frames, given certain number of users, is needed to provide reliability guarantees.

Approximations of the PLR for both the waterfall [7] and error floor [1] regimes can be found in the literature. ${ }^{1}$ While the former follows well the PLR for medium to high channel load values, the latter is meant for regions of low traffic load. Fig. 1 shows the packet loss rate results of a simulation with an IRSA frame of 10 slots together with both approximations, as a function of the channel load, i.e. the average number of users accessing per time slot. As an example of the IRSA access policy, we considered a scenario where three physical layer copies of the same packet are transmitted. As one can observe, the waterfall approximation has a good match for channel loads above 0.8. Nonetheless, the packet loss rate in this region is well above 0.5 (i.e. more than half of the packets cannot be correctly received), an unacceptable operating point for IIoT applications. Specifically, low PLR is one of the main requirements of IIoT, so the waterfall region is not further investigated in this work.

In the error floor regime, the EF approximation [1] can be adopted to predict the performance of the PLR. However, as clearly depicted in Fig. 1, the EF approximation rapidly diverges from the simulation already for rather small load values and does not provide a good prediction. It is evident that a tighter PLR approximation is needed for analyzing the performance of IRSA under very stringent latency requirements, enabling IIoT applications to benefit from such access policy.

Our contribution can be summarized as follows: we extend the EF approximation of [1]

\footnotetext{
${ }^{1}$ Another approximation of the PLR for IRSA can be found in [8]. Although being an interesting alternative, the results provided by the author appear to be less attractive compared to [1] in terms of tightness.
} 


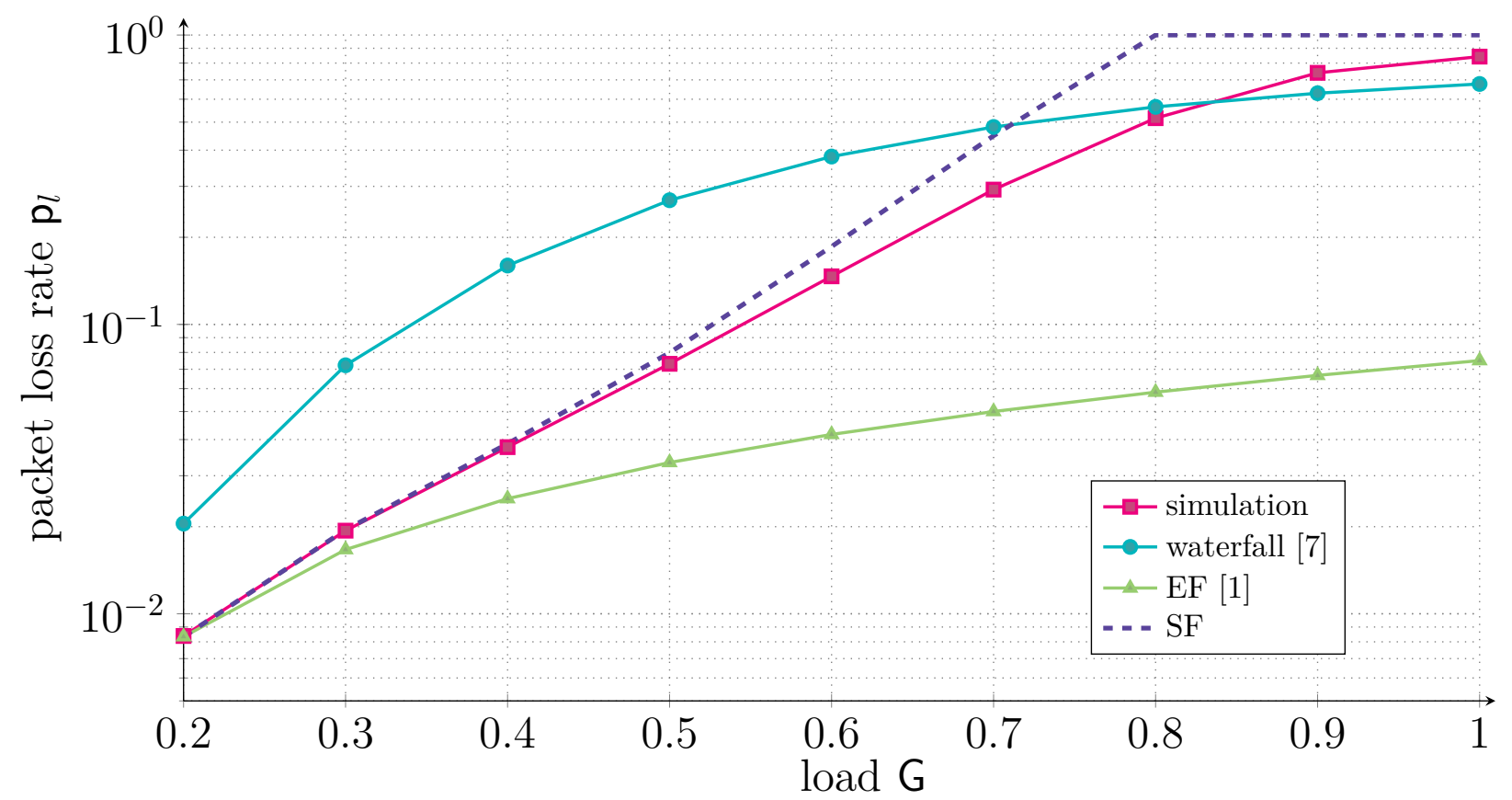

Fig. 1. An IRSA scenario with 10 slots. Three physical layer copies of the same packet are transmitted by each terminal. The packet loss rate as a function of the channel load is depicted. The unfitness of both the EF and the waterfall approximations is evident in this case.

going beyond the characterization of minimal stopping sets (STs). Specifically, we implement an integer-partition based algorithm to find the possible user and slot profiles of stopping sets. The algorithmic approach allows us to include more than 20,000 STs compared to ca. 60 STs considered in [1]. This of course incurs additional computational complexity. Two observations follow: on the one hand, the user degree distribution is normally defined offline while planning the network so that increase in computing time is a limited issue. On the other hand, compared to numerical simulation, the proposed approximation is still orders of magnitude faster.

The rest of the paper is organized as follows. The system model is introduced in Section II. Section III presents the key ingredients defining the stopping sets and describes the SF approximation of IRSA. The proposed SF approximation is compared with EF in Section IV, and Section V concludes the letter.

\section{System Model}

In this work, we consider a wireless uplink-only scenario where $m$ active users access the common medium according to the IRSA protocol. Sampling the user degree distribution $\Lambda(x)$, 
defined as

$$
\Lambda(x)=\sum_{\mathrm{d}=1}^{\mathrm{d}_{m}} \Lambda_{\mathrm{d}} x^{\mathrm{d}}, \quad \text { with } 0 \leq \Lambda_{\mathrm{d}} \leq 1 \forall \mathrm{d}, \quad \sum_{\mathrm{d}=1}^{\mathrm{d}_{m}} \Lambda_{\mathrm{d}}=1
$$

each transmitter determines how many copies it sends within the $n$ time slots composing the MAC frame. Transmission slots are selected uniformly at random and a maximum of $\mathrm{d}_{m}$ replicas are sent. ${ }^{2}$ Specifically, a user sends $d$ replicas of its packet with a probability of $\Lambda_{d}$ and each data unit has a pointer to the position of its copies. The channel load $\mathbf{G}=m / n$ is defined as the average number of users transmitting per time slot. We consider the collision channel model, i.e. collision-free replicas are decoded with probability 1 while data units involved in collisions are lost. At the receiver side, Successive Interference Cancellation (SIC) is employed to possibly resolve collisions iteratively removing replicas of users that experience at least one replica collision-free. We measure the performance in terms of PLR $\mathrm{p}_{l}$ which is defined as the number of non-recovered users divided by the total number of users transmitting.

\section{AnAlysis of Stopping Sets}

In [6], it has been observed that the received MAC frame can be conveniently visualized as a bipartite graph composed by two sets, users (UNs) and slots (SNs) connected by a set of edges identifying the slots where replicas belonging to each UN are transmitted. Thus, the interference cancellation iterative process can be tracked on the corresponding bipartite graph by identifying SNs with a single edge connected to them and resolving the connected user, i.e. removing all edges emanating by the UN. In fact, replicas transmitted collision-free can be correctly decoded, thanks to the collision channel model assumption. Unfortunately, not all collision patterns are resolved by resorting to SIC. Harmful collision patterns are named stopping sets (STs), ${ }^{3}$ and determine the packet loss rate. It is of utmost interest to characterize and analyze them, as we will do in the next Sections.

\section{A. Stopping Sets and their User, Slot Profiles}

Formally, a stopping set (ST) is a subgraph of the MAC frame in which no SNs has less than two emanating edges. Let us focus on a specific ST $\mathcal{S}$. In order to characterize the configuration of users, slots and their interlace within $\mathcal{S}$, we need to define:

\footnotetext{
${ }^{2}$ We assume that $d_{m}$ as a design parameter is selected such that $d_{m} \leq n$.

${ }^{3}$ The analogy with stopping sets of low parity check codes is evident and already highlighted in [9].
} 


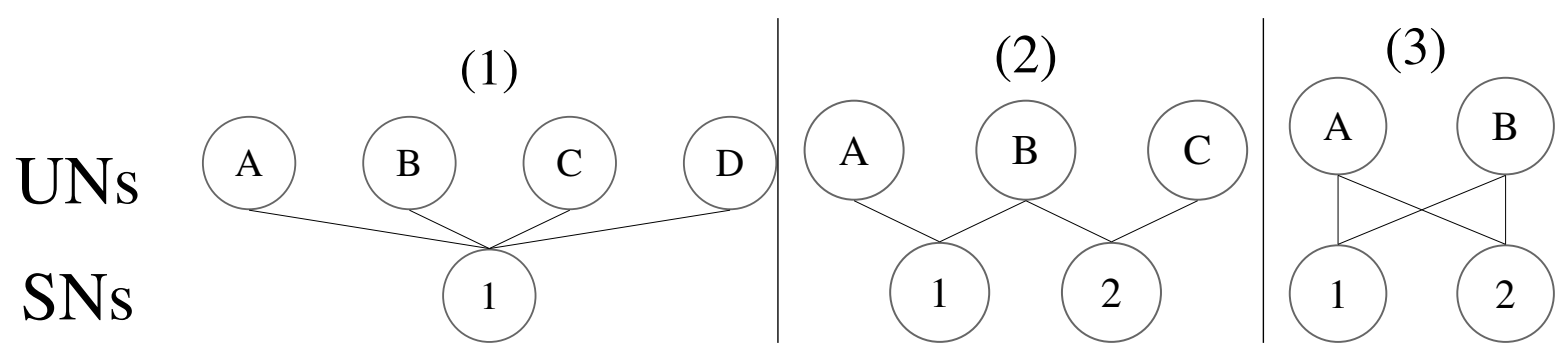

Fig. 2. The 3 possible STs (excluding the possible isomorphisms) with 4 edges.

\begin{tabular}{c|c|c}
\hline & $\boldsymbol{v}^{\mathcal{S}}$ & $\boldsymbol{\mu}^{\mathcal{S}}$ \\
\hline \hline$(1)$ & {$[4,0, \cdots, 0]$} & {$[0,0,0,1, \cdots, 0]$} \\
\hline$(2)$ & {$[2,1, \cdots, 0]$} & {$[0,2,0,0, \cdots, 0]$} \\
\hline$(3)$ & {$[0,2, \cdots, 0]$} & {$[0,2,0,0, \cdots, 0]$} \\
\hline
\end{tabular}

TABLE I

BN-SN PROFILES FOR STS WITH 4 EDGES.

- the user profile $v^{\mathcal{S}}=\left[v_{1}^{\mathcal{S}}, \cdots, v_{\mathrm{d}_{m}}^{\mathcal{S}}\right]$, where $v_{\mathrm{d}}^{\mathcal{S}}$ is the number of UNs in $\mathcal{S}$ with degree $\mathrm{d}$ and $v^{\mathcal{S}}$ is the $L^{1}$-norm of $\boldsymbol{v}^{\mathcal{S}}$, i.e. $v^{\mathcal{S}}=\left\|\boldsymbol{v}^{\mathcal{S}}\right\|_{1}$;

- the slot profile $\boldsymbol{\mu}^{\mathcal{S}}=\left[\mu_{1}^{\mathcal{S}}, \cdots, \mu_{m}^{\mathcal{S}}\right]$, where $\mu_{i}^{\mathcal{S}}$ is the number of SNs in $\mathcal{S}$ with degree $i$ and $\mu^{\mathcal{S}}$ is the $L^{1}$-norm of $\mu^{\mathcal{S}}$, i.e. $\mu^{\mathcal{S}}=\left\|\mu^{\mathcal{S}}\right\|_{1}$.

In Fig. 2 we present all possible STs with 4 edges, excluding the possible isomorphisms, where UNs are denoted by capital letters and SNs are denoted by numbers. Fixing the number of edges to 4 , there are 3 possible SN-BN profiles causing STs. Since a SN belonging to a stopping set has at least 2 emanating edges, there are a maximum of 2 and a minimum of $1 \mathrm{SN}$ involved. Configuration (1) sees 4 degree one UNs and 1 degree four SN, while ST (2) involves 2 degree one and 1 degree two UN and 2 degree two SNs. Finally, ST (3) encompass 2 degree two UNs and 2 degree two SNs. The corresponding user and slot profiles for the three configurations are summarized in Table I.

\section{B. Short Frame Packet Loss Rate Approximation}

Let us define $\mathcal{S}_{U}$ as the set of all possible stopping sets $\mathcal{S}$ and $\overline{\mathcal{S}} \subset \mathcal{S}_{U}$ a subset thereof. Given a user $u$, conditioned on $m$ users accessing the IRSA frame, the packet loss rate $\mathrm{p}_{l}(m)$ can be 
approximated as

$$
\begin{aligned}
\mathrm{p}_{l}(m) & =\operatorname{Pr}\left\{\bigcup_{\mathcal{S} \in \mathcal{S}_{U}} u \in \mathcal{S} \mid m\right\} \stackrel{(a)}{\leq} \sum_{\mathcal{S} \in \mathcal{S}_{U}} \operatorname{Pr}\{u \in \mathcal{S} \mid m\} \\
& \stackrel{(b)}{\approx} \sum_{\mathcal{S} \in \overline{\mathcal{S}}} \operatorname{Pr}\{u \in \mathcal{S} \mid m\}
\end{aligned}
$$

where (a) stems from the union bound, while (b) is the truncation to the subset $\overline{\mathcal{S}}$. Thus, we will consider only the most relevant stopping sets $\overline{\mathcal{S}}$ for the approximation of $\mathrm{p}_{l}(m)$, i.e. the STs with a limited number of UNs and SNs. It shall be noted that compared to [1], we will consider a larger set of STs. Focusing on a specific ST $\mathcal{S} \in \overline{\mathcal{S}}$ we can write [1]

$$
\operatorname{Pr}\{u \in \mathcal{S} \mid m\}=\frac{w\left(\boldsymbol{v}^{\mathcal{S}}, \boldsymbol{\mu}^{\mathcal{S}}\right)}{m} .
$$

We can now expand $w\left(\boldsymbol{v}^{\mathcal{S}}, \boldsymbol{\mu}^{\mathcal{S}}\right)$, the expected number of unresolved users belonging to the ST $\mathcal{S}$, as

$$
w\left(v^{\mathcal{S}}, \boldsymbol{\mu}^{\mathcal{S}}\right)=\alpha\left(\boldsymbol{v}^{\mathcal{S}}, m\right) \beta\left(\boldsymbol{\mu}^{\mathcal{S}}\right) \gamma\left(\boldsymbol{v}^{\mathcal{S}}, \boldsymbol{\mu}^{\mathcal{S}}\right) \delta\left(\boldsymbol{v}^{\mathcal{S}}, \boldsymbol{\mu}^{\mathcal{S}}\right)
$$

where

$$
\alpha\left(v^{\mathcal{S}}, m\right)=\frac{m !}{\left(m-v^{\mathcal{S}}\right) !} \prod_{\mathrm{d}=1}^{\mathrm{d}_{m}} \frac{\Lambda_{\mathrm{d}}^{\nu_{\mathrm{d}}^{\mathcal{S}}}}{v_{\mathrm{d}}^{\mathcal{S}} !} ; \quad \beta\left(\boldsymbol{\mu}^{\mathcal{S}}\right)=\left(\begin{array}{c}
n \\
\mu_{1}^{\mathcal{S}}, \ldots, \mu_{m}^{\mathcal{S}}
\end{array}\right) .
$$

In eq. (5), $\alpha$ represents the number of ways in which $v^{\mathcal{S}}$ users in the ST can be chosen from the total $m$ transmitting nodes, taking into account the selected user degree distribution. While $\beta$ is the total number of combinations of $\mu^{\mathcal{S}}$ slots belonging to the ST out of the total $n$. The function $\gamma\left(\nu^{\mathcal{S}}, \boldsymbol{\mu}^{\mathcal{S}}\right)$, representing the ratio between the number of STs that the user-slot profiles $\left(v^{\mathcal{S}}, \boldsymbol{\mu}^{\mathcal{S}}\right)$ can generate over the total number of graphs which $\boldsymbol{v}^{\mathcal{S}}$ can lead to, can be explicitly written as:

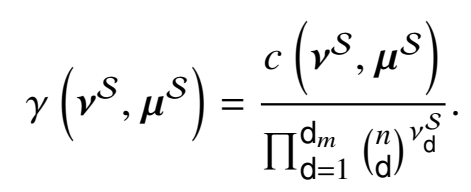

Here $c\left(\boldsymbol{v}^{\mathcal{S}}, \boldsymbol{\mu}^{\mathcal{S}}\right)$ is the number of isomorphic graphs with profiles $\left(\boldsymbol{v}^{\mathcal{S}}, \boldsymbol{\mu}^{\mathcal{S}}\right)$ (see also [1] for more details), which is computed numerically.

We define $\delta\left(\boldsymbol{v}^{\mathcal{S}}, \boldsymbol{\mu}^{\mathcal{S}}\right)$ (cf. [1]) as the probability of the remaining $m-v^{\mathcal{S}}$ users to connect to the stopping set $\mathcal{S}$ so that a larger ST $\mathcal{K} \supset \mathcal{S}$ does not appear. For example, a user that has transmitted a packet in a singleton slot, can connect to the stopping set $\mathcal{S}$. This case does not constitute a new stopping set as that user can easily be recovered thanks to the singleton slot. This 
probability is assumed to be 1 in [1] which in most of the cases overestimates the PLR. Next, we present a refined way to compute $\delta\left(v^{\mathcal{S}}, \boldsymbol{\mu}^{\mathcal{S}}\right)$. For any set $\mathcal{S}$, let us define $\mathrm{e}_{\mathcal{S}}=\sum_{\mathrm{d}=1}^{\mathrm{d}_{m}} \mathrm{~d} v_{\mathrm{d}}^{\mathcal{S}}$, as the number of edges in $\mathcal{S}$. Let $\mathcal{T}_{\mathcal{S}, \mathrm{e}_{m}}$ be the set of all STs containing $\mathcal{S}$ with all and only the $\mathrm{SNs}$ in $\mathcal{S}$ and with the number of edges not exceeding $\mathrm{e}_{m}>\mathrm{e}_{\mathcal{S}}$. The parameter $\mathrm{e}_{m}$ is introduced to keep the computational complexity of $\delta$ limited. We approximate $\delta\left(\boldsymbol{v}^{\mathcal{S}}, \boldsymbol{\mu}^{\mathcal{S}}\right)=1$ for $\mathrm{e}_{\mathcal{S}}=\mathrm{e}_{m}$ similarly to [1]. Instead, for all sets $\mathcal{S}$ with $\mathrm{e}_{\mathcal{S}}<\mathrm{e}_{m}$ we have

$$
\begin{array}{r}
\delta\left(v^{\mathcal{S}}, \boldsymbol{\mu}^{\mathcal{S}}\right) \approx 1-\sum_{\mathcal{K} \in \mathcal{T}_{\mathcal{S}, \mathrm{e}_{m}}} \alpha\left(v^{\mathcal{K}}-\boldsymbol{v}^{\mathcal{S}}, m-v^{S}\right) . \\
\tilde{\gamma}\left(\boldsymbol{v}^{\mathcal{K}}, \boldsymbol{v}^{\mathcal{S}}, \boldsymbol{\mu}^{\mathcal{K}}, \boldsymbol{\mu}^{\mathcal{S}}\right) \delta\left(v^{\mathcal{K}}, \boldsymbol{\mu}^{\mathcal{K}}\right) \\
\text { for } \mathrm{e}_{\mathcal{S}}<\mathrm{e}_{\mathcal{K}} \leq \mathrm{e}_{m}
\end{array}
$$

where $\alpha\left(v^{\mathcal{K}}-v^{\mathcal{S}}, m-v^{S}\right)$ in eq. (7) is the number of ways to select the users with the UN profile $v^{\mathcal{K}}-v^{\mathcal{S}}$ out of the $m-v^{S}$ not in $\mathcal{S}$, and with

$$
\tilde{\gamma}\left(v^{\mathcal{K}}, \boldsymbol{v}^{\mathcal{S}}, \boldsymbol{\mu}^{\mathcal{K}}, \boldsymbol{\mu}^{\mathcal{S}}\right)=\frac{\tilde{c}\left(\boldsymbol{v}^{\mathcal{K}}, \boldsymbol{v}^{\mathcal{S}}, \boldsymbol{\mu}^{\mathcal{K}}, \boldsymbol{\mu}^{\mathcal{S}}\right)}{\prod_{\mathrm{d}=1}^{\mathrm{d}_{m}\left(\begin{array}{l}
n \\
\mathrm{~d}
\end{array}\right)}\left(v_{\mathrm{d}}^{\mathcal{K}-v_{\mathrm{d}}^{\mathcal{S}}}\right)} .
$$

In eq. (8), $\tilde{c}\left(\boldsymbol{v}^{\mathcal{K}}, \boldsymbol{v}^{\mathcal{S}}, \boldsymbol{\mu}^{\mathcal{K}}, \boldsymbol{\mu}^{\mathcal{S}}\right)$ is the number of graphs that are isomorphic to $\mathcal{K}$ embedding the ST $\mathcal{S}$.

In order to compute $\delta$, one should define the maximum number of edges $\mathrm{e}_{m}$ considered for the ST. Starting from the ST with the maximum number of edges $\mathrm{e}_{m}$, where we assume $\delta=1$, one can proceed backwards removing one edge at a time till the ST of interest $\mathcal{S}$ as per eq. (7). It has to be stressed that the expression in eq. (7) is an approximation to the exact $\delta\left(\nu^{\mathcal{S}}, \boldsymbol{\mu}^{\mathcal{S}}\right)$; nonetheless, we show its tightness in the next section.

In order to compute the exact value of the packet loss rate, all possible stopping sets should be considered. Even for small frames, the number would rapidly become intractable, yielding unmanageable complexity. To limit it, we consider only STs that have up to $\mathrm{e}_{m}$ edges.

\section{EVALUATiON}

We consider a maximum of 10 users sending at least two and at most four replicas, i.e., $\mathrm{d}_{m}=4$. Thus, the total number of edges in the bipartite graph varies between 20 and 40 . We set $\mathrm{e}_{m}=20$ as a sufficient trade-off between complexity and tightness in the approximation. Note that in this way, only stopping sets that have up to 20 edges are considered. 


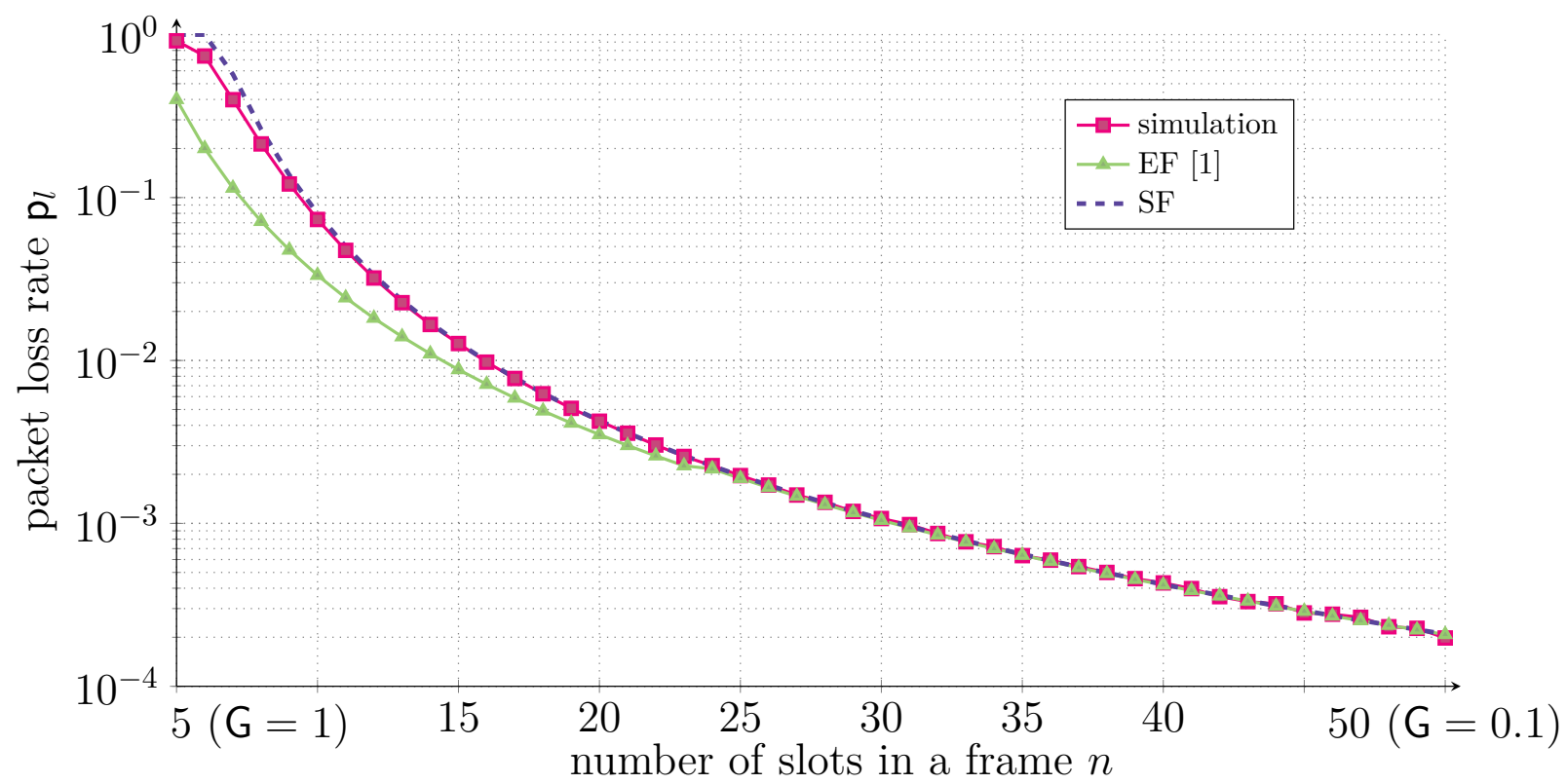

Fig. 3. Simulated packet loss rate vs. the EF approximation and the SF approximation in an IRSA scenario for $\Lambda(x)=x^{3}$ with a fixed number of users equal to 5 and variable number of slots.

The simulations are conducted in a discrete time simulator designed in MATLAB. ${ }^{4}$ For each frame, users generate a random number of replicas following the user degree distribution. Then each replica is transmitted uniformly at random in the IRSA frame. At the receiver side, upon storing the entire frame, SIC is performed to possibly resolve collision. At the end of the SIC process the packet loss rate (PLR) is measured. The results presented for each configuration are an average over $10^{6}$ runs.

As illustrated in Fig. 1, our SF approximation provides a maximum relative error of 0.54 for a channel load of $\mathrm{G} \leq 0.7$ compared to 0.83 of EF. In the unpractical range of PLR higher than $10^{-1}$, the approximation diverges. This is mainly due to the limited number of edges considered in the approximation. In fact, at high load, stopping sets with more than 20 edges occur more frequently. These results confirm that for the short frame regime our approximation is tighter than the EF approximation.

Fig. 3 aims at evaluating the SF approximation with variable load and illustrates an IRSA scenario with 5 users and a user degree distribution set to $\Lambda(x)=x^{3}$. The load is $1 \geq \mathrm{G} \geq 0.1$. Within the load range the SF approximation leads to a maximum relative error of 0.43 , much

\footnotetext{
${ }^{4}$ We plan to make the simulator open-source available for the camera ready version of the paper.
} 


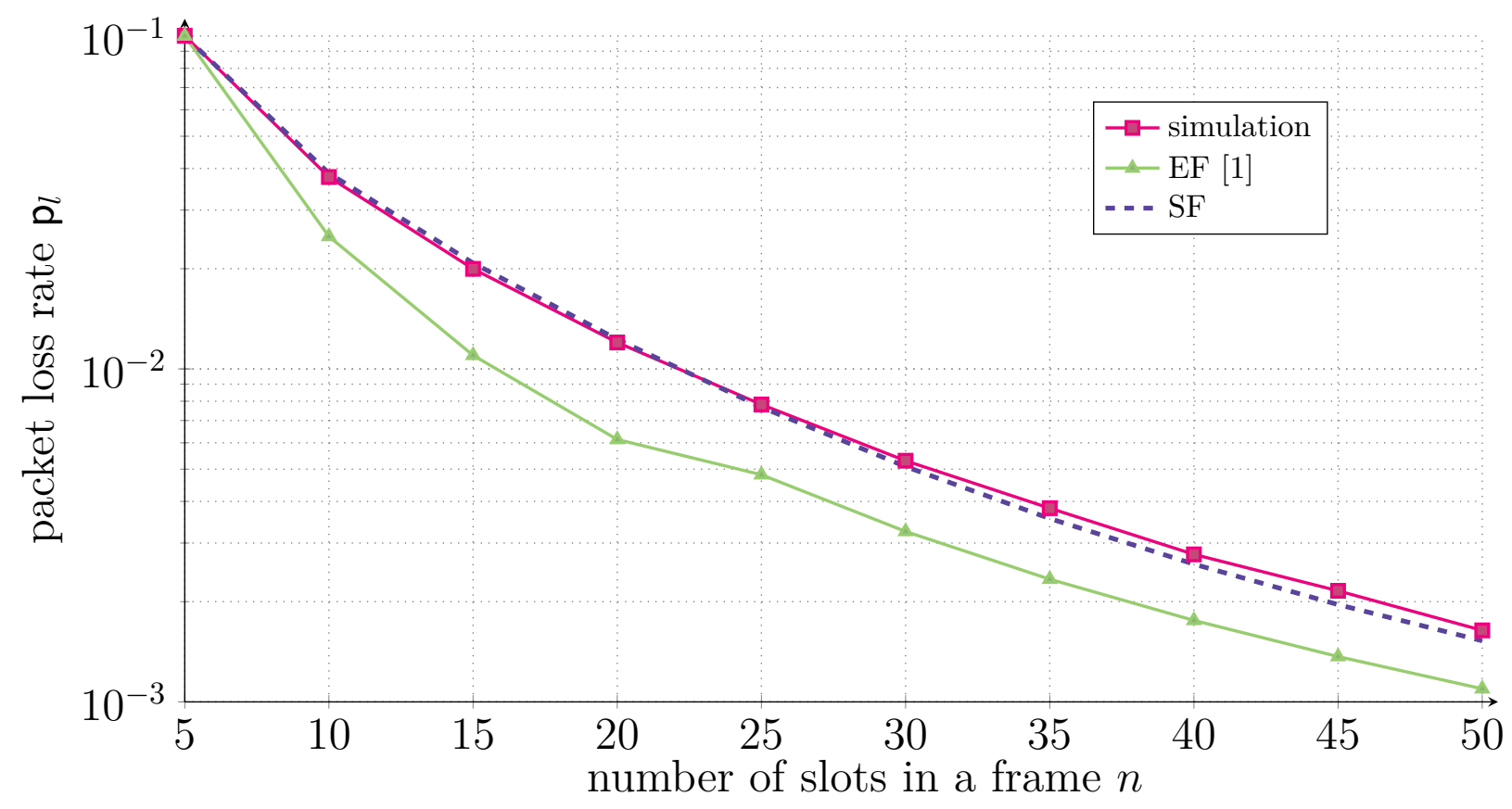

Fig. 4. Packet loss rate computed with the EF approximation, the SF approximation and via numerical simulations. The user degree distribution is $\Lambda(x)=x^{3}$ with $\mathrm{G}=0.4$ and a variable number of slots.

smaller than 0.73 , the one achieved by the EF. For a channel load of 0.5 or below, the maximum relative error is impressively reduced to 0.08 for the SF compared to 0.61 for the EF. Overall, SF approximation outperforms the EF approximation with variable load and with a fixed number of users.

Fig. 4 aims at evaluating the SF approximation with a fixed load and variable number of slots and users. We set the load $G=0.4$ users per slot and the user degree distribution to $\Lambda(x)=x^{3}$. The frame size is in the range $n \in\{5, \ldots, 50\}$ and the number of users varies in $m \in\{2, \ldots, 20\}$. In this second setup, the EF approximation shows a maximum relative error of 0.49 that can be reduced to 0.09 with the SF approximation.

The presented evaluations have shown a tighter match of the proposed SF approximation compared to the EF. In order to leverage such benefits, we now exploit the SF to optimize the user degree distribution. The target is to minimize the PLR $\mathrm{p}_{l}$ for a given number of users $m$ and a fixed frame size $n$. To this end we fix $m=5$ users and $n=10$ slots. In order to have a tractable problem, we limit ourselves to a degree distribution $\Lambda(x)=\Lambda_{2} x^{2}+\left(1-\Lambda_{2}\right) x^{4}$, where $\Lambda_{2}$ is our optimization variable. Recall that $\Lambda_{2}$ is the probability that a user transmits two replicas within the MAC frame, thus $0 \leq \Lambda_{2} \leq 1$. In our formulation, $\left(1-\Lambda_{2}\right)=\Lambda_{4}$, the probability that 


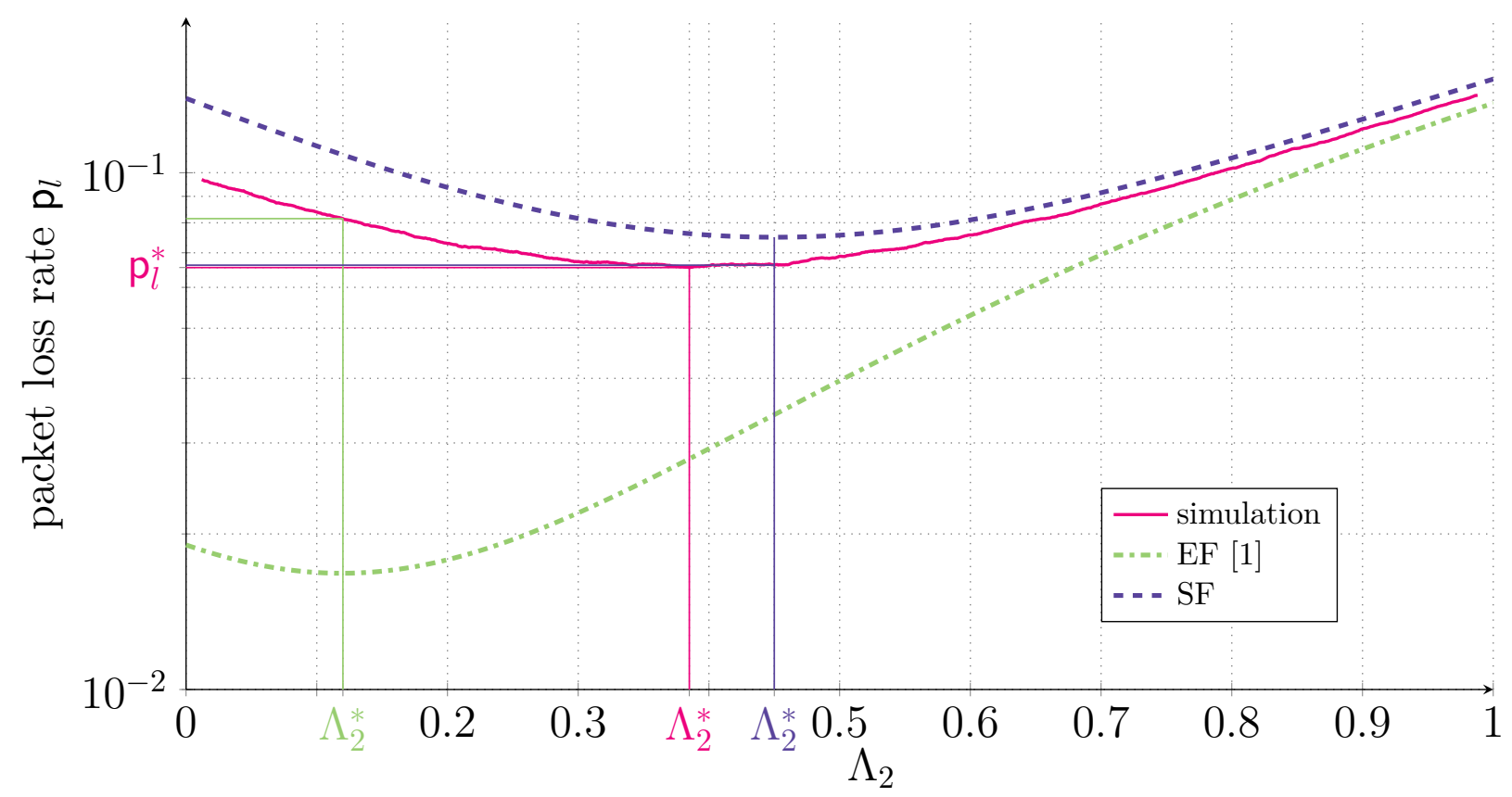

Fig. 5. Packet loss rate computed with the EF approximation, the SF approximation and by numerical simulations as a function of $\Lambda_{2}$. The scenario assumes $n=10$ slots, $m=5$ users per frame and $\Lambda(x)=\Lambda_{2} x^{2}+\left(1-\Lambda_{2}\right) x^{4}$.

a user sends four replicas. The simulation results with EF approximation and SF approximation are shown in Fig. 5. The probability $\Lambda_{2}$ is depicted on the x-axis, while y-axis represents the packet loss rate. The blue, the black and the red lines correspond to the EF approximation, the SF approximation, and the simulation, respectively. As expected, the SF approximation follows the simulation closely. Observe that we can numerically evaluate the extremal point for both approximations. Since the curves are convex, determining the extremal point corresponds to minimize the PLR. Once found the $\Lambda_{2}$ that minimizes the PLR, we can compare it with the minimum obtained via Monte Carlo simulations. For the SF approximation, the minimum PLR is 0.0662 , achieved for $\Lambda_{2}^{*}=0.45$. The minimum PLR with the EF approximation results in $\mathrm{p}_{l}=0.0815$, which is almost $24 \%$ more than the value with $\mathrm{SF}$ and is found for $\Lambda_{2}^{*}=0.12$. The simulated minimum is $\mathrm{p}_{l} \approx 0.0655$ that is only $10 \%$ lower than PLR achieved by SF approximation and is obtained by $\Lambda_{2}^{*}=0.385$.

\section{CONCLUSION}

In this paper, we introduced the short-frame (SF) approximation for the PLR in IRSA. Targeting IIoT applications with stringent latency constraints, an extension of the analysis in 
[1] is provided. Not only a larger set of stopping sets is included in the analysis, but also a refinement is proposed. Along with the definition of the slot profile also the inclusion of nonminimal stopping sets helps improving the approximation. Increased tightness of up to one order of magnitude for frames till 50 slots is shown via numerical results. The user degree distribution minimizing the PLR can be easily and reliably obtained with the SF even for very short frames, where alternatives become loose. Additionally, an open-source algorithm is provided for the SF approximation and can be exploited for more precise results by considering more stopping sets. An interesting future direction is to consider real traffic profiles while embedding the user degree optimization relying on the proposed PLR approximation.

\section{REFERENCES}

[1] M. Ivanov, F. Brannstrom, A. G. i Amat, and P. Popovski, "Broadcast coded slotted aloha: A finite frame length analysis," IEEE Transactions on Communications, vol. 65, no. 2, pp. 651-662, 2017.

[2] E2E-aware Optimizations and advancements for Network Edge of 5G New Radio (ONE5G), "Deliverable D2.1: Scenarios, KPIs, use cases and baseline system evaluation,” Tech. Rep., nov 2017. [Online]. Available: https://one5g.eu/

[3] “3GPP TS 38.331 V15.2.1: NR; Radio Resource Control (RRC) protocol specification,” 2018.

[4] M. Berioli, G. Cocco, G. Liva, and A. Munari, Modern Random Access Protocols. Now Foundations and Trends, 2016.

[5] H. M. Gürsu, M. C. Moroğlu, M. Vilgelm, F. Clazzer, and W. Kellerer, "System level integration of irregular repetition slotted aloha for industrial iot in 5g new radio," in Personal, Indoor and Mobile Radio Communications (PIMRC 19). IEEE, 2019.

[6] G. Liva, "Graph-based analysis and optimization of contention resolution diversity slotted aloha," IEEE Transactions on Communications, vol. 59, no. 2, pp. 477-487, 2011.

[7] A. G. i Amat and G. Liva, "Finite-length analysis of irregular repetition slotted aloha in the waterfall region," IEEE Communications Letters, vol. 22, no. 5, pp. 886-889, 2018.

[8] E. Paolini, "Finite length analysis of irregular repetition slotted aloha (irsa) access protocols," in 2015 IEEE International Conference on Communication Workshop (ICCW). IEEE, 2015, pp. 2115-2120.

[9] M. Ivanov, F. Brannstrom, A. G. i Amat, and P. Popovski, "Error floor analysis of coded slotted aloha over packet erasure channels," IEEE Communications Letters, vol. 19, no. 3, pp. 419-422, 2015. 